\title{
Imaging Appetite
}

Blanca Lizarbe ${ }^{1}$, Ania Benítez ${ }^{1,2}$, Manuel Sánchez-Montañés ${ }^{2}$, Luis F. Lago-Fernández ${ }^{2}$, María Luisa Garcia-Martin ${ }^{3,4}$, Pilar López-Larrubia ${ }^{1}$ and Sebastián Cerdán ${ }^{1}$

${ }^{1}$ Instituto Investigaciones Biomédicas "Alberto Sols" CSIC-UAM, c/ Arturo Duperier 4, Madrid 28029, Spain

${ }^{2}$ Departamento de Ingeniería Informática, Escuela Politécnica Superior, Universidad Autónoma de Madrid, Cantoblanco, Madrid 28049.

${ }^{3}$ Resonancia Magnética Nuestra Señora del Rosario, Príncipe de Vergara 56, 28006 Madrid (Spain).

${ }^{4}$ Andalusian Centre for Nanomedicine and Biotechnology (BIONAND), Málaga, Spain

Corresponding author: Prof. Sebastián Cerdán

Instituto de Investigaciones Biomédicas "AAlberto Sols" CSIC/UAM

c/ Arturo Duperier 4

Madrid 28028

Telf. 0034 91-5854444

Fax: 003491-585-4401

E-mail: scerdan@iib.uam.es

Running title: Imaging appetite

(Abstract 250 words, Introduction 511 words, Discussion 797 words, 4 Figures)

Key words: Appetite regulation, Functional Imaging, Cerebral Activation, Diffusion Weighted MRI, Image Analysis

Abbreviations: $D_{\text {slow: }}$ Slow diffusion coefficient, $D_{\text {fast }}$ : Fast diffusion coefficient, FDP: Fast diffusion phase, LDA: Linear Discriminant Analysis, SDP: Slow diffusion phase,

\section{Acknowledgements}

Authors are indebted to Dr. Diana Quiñones Tapia for expert neuroradiological assistance during the human studies and to Mr. Javier Pérez CSIC, for careful, professional drafting of the illustrations.

This work was supported in part by grants SAF-2008-01327 and SAF2011-23622 to SC, grant CTQ-2010-20960-C02-02 to PLL, grants S-BIO-2006-0170 and S2010/BMD-2349 to S.C., PLL and MSM and grant CAM/UAM (CCG10-UAM/TIC-5864) to LFLF. BL and ABSDC held predoctoral fellowships from the Spanish Ministry of Science and Technology (BES 2009027615) and the Spanish Agency for International Cooperation and Development. 


\section{Abstract}

Hypothalamic appetite regulation is a vital homeostatic process underlying global energy balance in animals and humans, its disturbances resulting in feeding disorders with high morbidity and mortality. The objective evaluation of appetite remains difficult, very often restricted to indirect measurements of food intake and body weight. We report here, the direct, non invasive visualization of hypothalamic activation by fasting using Diffusion Weighted Magnetic Resonance Imaging, in the mouse and human brains. The brain of fed or fasted mice or humans were imaged at 7 or 1,5 Tesla, respectively, by diffussion weighted magnetic resonance imaging using a complete range of $b$ values $\left(10<b<1800 \mathrm{~s} . \mathrm{mm}^{-2}\right)$. The diffusion weighted image data sets were registered and analyzed pixel by pixel using a biexponetial model of diffusion, or a model-free Linear Discriminant Analysis approach. Biexponetial fittings revealed statistically significant increases in the slow diffusion parameters of the model, consistent with a neurocellular swelling response in the fasted hypothalamus. Linear Discriminant Analysis was able to classify successfully the diffusion data sets from mice and humans between fed and fasted states, suggesting intrinsic differences supplementary to those detected by the alternative biexponential model. Our results are consistent with increased glutamatergic neurotransmission during orexigenic firing, a process resulting in increased ionic accumulation and concomitant osmotic neurocellular swelling. This swelling response,is spatially extendable through surrounding astrocytic networks until it becomes MRI detectable. Present findings open new avenues for the direct, non invasive, evaluation of appetite disorders and other hypothalamic pathologies helping potentially in the development of the corresponding therapies.

Key words: Appetite regulation, Functional Imaging, Cerebral Activation, Diffusion Weighted MRI, Image Analysis 


\section{Introduction}

The appetite impulse originates in the brain from an imbalance in the systemic and intrahypothalamic mechanisms controlling food intake and energy expenditure (Morton et al., 2006). Following a meal, increased insulin and leptin levels induce an anorexigenic response consisting in a reduction in food intake and an increase in energy expenditure, whereas in fasting periods decreased plasma levels of leptin and insulin promote increased food intake and energy expenditure. These systemic and intrahypothalamic neuroendocrine signals interact mainly in the Arcuate and Paraventricular nuclei, modifying the balance between the activity of orexigenic neurons, releasing Agouti Related Peptide (AgRP) or Neuropeptive Y (NPY), and anorexigenic neurons releasing Proomileanocortin (POMPCT) or Cocaine Anfetamine Receptor Transcript (CART) (Coll et al., 2007). In addition to neuroendocrine signalling, appetite stimulation is known to involve hypothalamic increases in glutamatergic and gabaergic neurotransmissions (van den Pol et al., 1990; Delgado et al., 2011). Moreover, (Spanswick, 2012), reported recently that the excitatory synaptic control of AgRP neurons is regulated by fasting and hormones and that increased glutamatergic activity is a necessary requirement for a physiological response to fasting (Yang et al., 2011; Liu et al., 2012).

Despite the progress obtained in the interpretation of the molecular events underlying the fasting response, the direct evaluation of appetite regulation in the brain still remains difficult, very often limited to indirect measurements of food intake and body weight. On these grounds, the implementation of non invasive neuroimaging methodologies for the evaluation of appetite entails considerable relevance. A variety of neuroimaging tools have been proposed including mainly positron emission tomography (PET) and functional Magnetic Resonance Imaging (fMRI) methods (Carnell et al., 2011). PET studies provide information on cerebral activation by detecting the emitted positrons derived from the increased uptake of ${ }^{18} \mathrm{~F}$-deoxiglucose, an event revealing the metabolic coupling between glucose uptake, blood flow and neuronal activity in the hypothalamus during feeding-related stimuli (Tataranni et al., 1999; Gautier et al., 2000). Blood Oxygenation Level Dependent (BOLD) fMRI, infers regional neuronal activity from the changes in magnetic susceptibility that take place in the transition between oxygenated and deoxygenated hemoglobin, occurring after increased oxygen delivery to activated neurons in the hypothalamus of rats and humans (Matsuda et al., 1999; Mahankali et al., 2000). Finally, 
Manganese Enhanced Magnetic Resonance Imaging (MEMRI), uses manganese ion accumulation as a surrogate marker of the increased calcium movements occurring during neuronal activation. MEMRI has revealed, the time course of hypothalamic activation, as a response to the systemic administration of different orexigenic or anorexigenic peptides (Parkinson et al., 2009). Notably, these previous approaches are not devoid of limitations to investigate hypothalamic physiology in animals and man, mainly derived from their reduced spatial and temporal resolution in the PET and BOLD fMRI approaches, and the potential neurotoxicity of $\mathrm{Mn}^{2+}$, in the MEMRI technique. To overcome these limitations, we propose here the use of functional Diffusion Weighted Imaging fDWI (Le Bihan, 2003) a novel functional approach improving the spatial and temporal resolution provided earlier by PET or BOLD and avoiding the use of the potentially toxic doses of manganese precluding its use of MEMRI in humans.

\section{Materials and Methods}

Experimental models. The experimental protocols used in this study were approved by the appropriate institutional committees and met the guidelines of the appropriate government agency. Experiments with animals were carried out using healthy adult male C57BL/6 mice $(n=6,25 \pm 4 g)$. Each animal was investigated in two successive experimental conditions both receiving drinking water ad libitum; "fed"; receiving normal mice chow diet (A04 http://www.safediets.com/eng/home/home.html, SAFE Augy, France, $2900 \mathrm{Kcal} / \mathrm{Kg}$ ), and "fasted"; 48 hours after complete food removal. In all small animal imaging experiments, anesthesia was initiated in a plexiglass induction box (Isofluorane $2 \% / 99.9 \% \mathrm{O} 2,1 \mathrm{~mL} / \mathrm{min}$ ) and maintained during the imaging time with a nose mask (Isofluorane 1\%/O2 99,9\% mixture, $1 \mathrm{~mL} / \mathrm{min}$ ). Anesthetized animals were placed in a water heated probe, which maintained the core body temperature at approximately $37^{\circ} \mathrm{C}$ during scanning. The physiological state of the animal during the imaging process was monitored by the respiratory rate and body temperature using a Biotrig physiological monitor (Bruker Biospin, Ettlingen, Germany).

Human participants in the study were six healthy male volunteers, aged $24-33$. Conditions for the participation were: (1) Healthy clinical trajectory without familiar antecedents of obesity, diabetes or other endocrine disorders; (2) Body Mass Index (BMI) of 18.5-25, corresponding to 
normal body weight; (3) volunteers were required to follow a balanced diet $(2000-2500 \mathrm{cal} / \mathrm{Kg})$ during seven days previous to the basal image acquisitions, with no drinks other than water ad libitum, no medication or abnormal exercise. Each Volunteer was imaged in two successive conditions; first, "fed", after one week of a balanced diet and second "fasted", 24 hours after food deprivation. Blood samples from the median cubital vein were drained before the "fed" and "fasted" image acquisitions and analyzed for routine biochemical parameters T3, T4, TSH and insulin levels.

MRI sequences. The Magnetic Resonance Imaging (MRI) experiments with mice were performed on a 7.0-T horizontal-bore superconducting magnet (Bruker Pharmascan, Bruker Medical Gmbh, Ettlingen, Germany) equipped with a ${ }^{1} \mathrm{H}$ selective birdcage resonator of $23 \mathrm{~mm}$ and a $90 \mathrm{~mm}$ of diameter gradient insert (36 gauss $/ \mathrm{cm})$. Imaging data were acquired using Hewlett-Packard console running Paravision 4.0 software (Bruker Medical Gmbh, Ettlingen, Germany) operating on a Linux environment.

\section{$<$ Figure 1, near here>}

Figure 1 provides an overview of the acquisition and image analysis approaches implemented in this study and the regions of interest investigated, as illustrated for the mouse brain. A collection of diffusion weighted images of the fed and fasted mouse brain was obtained (left panel) and analyzed using either a biexponential diffusion model (upper right panels) or a model-independent Linear Discriminant Analysis approach (lower right panels). Briefly, at least six diffusion weighted images (DWI) were acquired across an axial plane containing the hypothalamus (Paxinos, 2001) (bregma,-1.46 mm) with b values of $300,600,900,1200,1500$ and $1800 \mathrm{~s} . \mathrm{mm}^{-2}$, with the diffusion gradient oriented along three orthogonal directions; LeftRight (L-R), Antero-Posterior (A-P) and Head-Feed (H-F). Additional lower b values were also acquired where indicated, including up to six $b$ weightings in the range $10<b<100 \mathrm{~s} \cdot \mathrm{mm}^{-2}$, to emphasize contributions of flow and perfusion (Le Bihan, 2008). Acquisition parameters were: repetition time $(T R)=3000 \mathrm{~ms}$, echo time $(T E)=51 \mathrm{~ms}$, four shot EPI readout, averages $(A v)=3$, $\Delta=20 \mathrm{~ms}, \delta=4 \mathrm{~ms}$, field of view $(\mathrm{FOV})=35 \mathrm{~mm}$, acquisition matrix $=128 \times 128$, corresponding to an in-plane resolution of $296 \times 296 \mu \mathrm{m}^{2}$, slice thickness $1.5 \mathrm{~mm}$ and number of slices 3 . Total acquisition time was 13 min $36 s$. 
$T_{2}$-weighted $\left(T_{2 w}\right)$ spin echo anatomical images were acquired using the rapid acquisition with relaxation enhancement (RARE) sequence in the same axial plane with the following parameters: TR=3200 ms, TE $=60 \mathrm{~ms}, \mathrm{RARE}$ factor $=8, \mathrm{Av}=3, \mathrm{FOV}=3.8 \mathrm{~cm}$, acquisition matrix $=256 \times 256$ corresponding to an in-plane resolution of $148 \times 148 \mu \mathrm{m}^{2}$, slice thickness $=1.5 \mathrm{~mm}$ and number of slices $=3$.

The Magnetic Resonance Imaging (MRI) experiments with human volunteers were performed in the Magnetic Resonance Unit of the Hospital Nuestra Señora del Rosario (Madrid, Spain), using a GE Medical Systems 1.5-T horizontal-bore superconducting magnet, equipped with a ${ }^{1} \mathrm{H}$ quadrature head resonator. Prior to the imaging experiments, volunteers signed up an informed consent and confidentiality document. Image acquisitions were medically supervised by the neuroradiology staff of the clinic. Multi b-value diffusion weighted images where acquired using $6 \mathrm{~b}$ values $\left(200,400,600,800\right.$ and $\left.1200 \mathrm{~s} / \mathrm{mm}^{2}\right) . T_{2}$-weighted $\left(T_{2 w}\right)$ spin echo anatomical images were acquired in every subject in the same plane as the DWI, using a rapid acquisition with Fast Relaxation Fast Spin Echo (FRFSE) sequence in coronal orientations. Acquisition parameters were: $\mathrm{TR}=3200 \mathrm{~ms}, \mathrm{TE}=90 \mathrm{~ms}$, Echo number=1, Echo Train Length=8, Av=4, FOV $=240 \mathrm{~mm}$, acquisition matrix $=512 \times 512$, corresponding to an in-plane resolution of $468.7 \times$ $468.7 \mu \mathrm{m}^{2}$, slice thickness $=3 \mathrm{~mm}$.

Image analysis. Images acquired with the small animal or human scanners were transformed in DICOM format and exported to external HP Z-400 Workstations operating on the Windows7 environment (http://emea.microsoftstore.com/es/es-ES/Microsoft/Windows/Windows7). Image analysis was performed as described below, using Statistical Parametric Mapping Sofware (SPM, http://www.fil.ion.ucl.ac.uk/spm/software) and a collection of in house developed programs (Matlab v7, The Mathworks, Nattick, MA, USA) for non linear model fitting and classification of diffusion weighted images using Linear Discriminant Analysis.

Diffusion Model. We used the biexponential model of attenuation of the DWI signal (Niendorf et al., 1996), as described by the expression;

$$
S(b) / S(0)=S D P \cdot \exp \left(-b \cdot D_{\text {slow }}\right)+F D P \cdot \exp \left(-b \cdot D_{\text {fast }}\right)
$$


where, $S(b)$ and $S(0)$ represent the individual pixel intensities in the presence and absence of diffusion gradient, $b$ indicates the diffusion weighting factor $\left(s \cdot \mathrm{mm}^{2}\right)$, SDP represents the Slow Diffusion Phase containing water molecules moving with a slow diffusion coefficient $D_{\text {slow, }}$, and FDP refers to the Fast Diffusion Phase containing the water molecules moving with a fast diffusion coefficient $D_{\text {fast. }}$. The addition of diffusion phases represents the total water molecules contributing to the signal decay $(\mathrm{SDP}+\mathrm{FDP}=1)$.

Parameter fitting and Statistical Analysis. Parameters of the biexponential model were determined independently for each voxel and direction in the two feeding states, using the non linear least-squares fitting Trust-Region algorithm (Matlab 2007b, The Mathworks, Nattick, MA, USA), customized to limit parameter ranges and optimize the goodness of fit. In particular, the goodness of fit $\left(\mathrm{r}^{2}\right)$ was restricted to be higher than 0.95 to guarantee an optimal fitting. Parameters are presented as mean \pm standard deviation within the two considered ROls (hypothalamus and an area of the cortex) in each animal or human. Statistical comparisons between the fed and fasted conditions were made constructing a cumulative, representative $\mathrm{ROI}$ that grouped the contributions of the six individual ROIs from each mouse or human. The statistical analysis was made using unpaired Student's test (as shown in Figs 2 and 3 ) as processed with SPSS (IBM Statistical package for the social sciences, 2000, http://www.spss.com).

Model-free DWI analyses. We also implemented a model-free classification algorithm, based on Fisher Linear Discriminant Analysis (Hastie et al., 2001). This model-free approach, allowed to investigate whether the DWI images could be automatically classified into the "fed" and "fasted" groups, without the constrains imposed by the biexponential model. LDA constructs a projection of the original data onto a low dimensional subspace trying to maximize the separation amongst the different classes. When there are only two classes, as in the present case (fed/fasted), this subspace is one-dimensional. Hence, the projection may be interpreted as an Appetite Index that can be used to discriminate between the two conditions (see Figure 1). 


\section{Results}

$<$ Figure 2, near here $>$

Figure $2 \mathrm{~A}$ shows the anatomical localization of MRI sections used to localize the hypothalamus in the mouse brain and the directions of diffusion measurements. Hypothalamic activation after 48 hours of fasting is conveniently detected by fDWI as a shift to red in the fasted condition, in the diffusion maps shown in Figures 2B (left images). The upper images show the hypothalamic SDP maps (A-P direction) in the fed and fasted conditions, superimposed on the corresponding anatomical $T_{2}$-weighted $\left(T_{2 w}\right)$ images from the whole brain. These hypothalamic SDP maps are shown enlarged in the corresponding lower images. The shift to orange and red, revealing increased SDP, is clearly detected in the fasted condition. Figures $2 \mathrm{~B}$ (right bar graphs) summarize the mean values, standard deviations and comparative statistical significances of the diffusion parameters (SDP, $D_{\text {slow }}$ and $D_{\text {fast }}$ ), from six mice under the fed and fasted conditions, investigated in three orthoghonal directions (L-R, A-P and H-F). SDP and $D_{\text {slow }}$ increased significantly with fasting $(p<0.01$ and $p<0.001)$ in the $L-R$ and A-P directions, whereas $D_{\text {fast }}$ decreased in the A-P and H-F orientations $(p<0.01)$.

The impact of this fasting paradigm was investigated additionally in the cerebral somatosensory cortex of the same animals, obtaining a different, albeit significant, diffusional response (Figures 2C). In particular, subsidiary effects of fasting become significant as reflected by the red-shift in the A-P SDP parameter of fasted animals (Figures 2C, upper left images), reflecting most probably, motor activations related to the feeding impulse. Enlarged SDP maps with improved resolution in this region are shown below (Figures 2C, lower left images), Mean values, standard deviations and statistical significance of the different diffusion parameters are shown as bar graphs in the right panels of Figure $2 C$. SDP and $D_{\text {slow }}$ increase significantly $(p<0.05)$ in the A-P direction and $D_{\text {fast }}$ decreases $(p<0.05)$ in the $L-R$ measurements.

Notably, the model independent analysis of the diffusion weighted data sets using Linear Discriminant Analisys (LDA) (Figures 2D,) was also able to classify successfully all mice investigated between the fed and fasted states. This indicaties that the intrinsic differences between fed and fasted brains may be detected even automatically, independently of the diffusion model used. The left image shows how SPM processing detected, as activated areas 
by the fasting paradigm, an area including the hypothalamus (yellow arrow) and an area of subsidiary vascular activation with the carotid arteries and the jugular veins (blue arrow). Taken together these evidences reveal a highly complex cerebrovascular response to the fasting paradigm involving not only hypothalamic activation but also an additional vascular adaptation. LDA was also applied to detect the most different pixels between the fed and fasted image data sets of every mouse. Results from a representative mouse are illustrated in the central and right panels. The Fisher's LDA projection (Appetite Index, central pannel) separates optimally the pixels of the fed (blue) and fasted (red) states. The pixels that are most representative of each state, located at the edges of the histogram $(<5 \%$ or $>95 \%)$, are shown in right panels, superimposed on the corresponding $T_{2}$ images. Using a leave one out cross validation strategy, it was possible to classify correctly, as fed or fasted, the image data sets from all mice investigated, when using the diffusion data sets acquired with eleven b values (six high $b$ and five low b). However, only five mice out of six mice investigated, were classified correctly between fed and fasted states, using only the six higher $b$ value data sets. This finding suggests that the microvascular contributions emphasized by the low $b$ values are needed to obtain the optimal discriminant power required to classify correctly the images of every mouse between the fed and fasted states.

$<$ Table 1, near here $>$

Table 1 shows the results for the blood samples taken from the cubital vein of the human volunteers before the "fed" and "fasted" image acquisitions. Blood samples were analyzed for routine biochemical and endocrine parameters including; glucose, cholesterol, triglycerides, HDL, LDL, T3, T4, TSH and insulin. Values of these parameters fell within the normal clinical range both in the "fed" and "fasted" conditions for every individual.

$<$ Figure 3, near here $>$

Figure $3 \mathrm{~A}$ shows the localization of $\mathrm{MRI}$ sections containing the hypothalamus in a human brain model and the directions of our DWI measurements. The diffusion maps shown in Figures 3B (left images) show that fDWI visiblydetects the hypothalamic activation induced by 24 hours of fasting. The upper images show the hypothalamic SDP maps (H-F direction) in the fed and fasted conditions, superimposed on the corresponding anatomical $T_{2 w}$ images from the whole 
brain. These hypothalamic diffusion maps are shown enlarged in the corresponding lower images. The fasted condition is clearly detected by a shift to orange and red of the SDP colour map, revealing a significant increase in SDP. Figures 3B (right bar graphs) summarize the mean values, standard deviations and comparative statistical significances of the diffusion parameters (SDP, $D_{\text {slow }}$ and $D_{\text {fast }}$ ) fitted as described in the methods section. We investigated six healthy volunteers under the fed and fasted conditions, monitoring the diffusion of water molecules in three orthoghonal directions (L-R, A-P and H-F). As in the ouse brain, we found also a significant increase of SDP values with fasting $(p<0.05)$. However, the human brain did not show significant cortical activation associated to the fasting paradigm (Figures $3 \mathrm{C}$ right bar graphs).

LDA of the human hypothalamic data set was also able to separate successfully the diffusion weighted images from the fasted and fed conditions (Figures 3D), implying again that the intrinsic differences between fDWI data sets in the two feeding conditions, are independent of the diffusion model used. A distribution of the fed and fasted projections from the hypothalamus of a representative subject is illustrated in the histogram (Appetite Index, left panel). The images in the upper right panels show enlarged, the hypothalamic areas that best discriminate the fed (blue pixels) and fasted (red pixels) groups and the corresponding localizations in anatomical T2 weighted images of the human brain (lower panels). An evaluation of the accuracy of classifications between fed and fasted states, as performed with the leave one out strategy, classified correctly the imaging data sets of five out six subjects, the same percentage obtained in the mouse brain images acquired with six b values under similar conditions.

\section{Discussion}

We report here that fasting results in significant changes in the diffusion parameters of cerebral water in mice and humans providing a novel approach for the objective, non-invasive, fully translational evaluation of the feeding impulse in vivo. Our data are consistent with previous applications of fDWI to cerebral activation studies, in particular of visual activation in humans (Le Bihan et al., 2006) and with in vitro DWI measurements of induced neuronal activity (Flint et al., 2009). In these cases, neuronal activation was associated to increments of the slow 
diffusion parameters in the activated areas, a finding proposed to reveal a relative redistribution of water molecules between the fast (FDP) and slow (SDP) diffusion phases, most probably reflecting, activation-induced increases in neurocellular volume (swelling) (Le Bihan et al., 2006).

The interpretation of the physiological or pathological changes in water diffusion parameters in the in vivo brain, has been a matter of debate in the last decades. Early physiological interpretations described the existence of two dynamically different diffusion phases, fast and slow, attributed initially to the extracellular and intracellular environments (Garcia-Martin et al., 2001), respectively. Further studies showed that the volume fractions of the intra- and extracellular phases predicted by DWI did not match those determined histologically (Niendorf et al., 1996; Sehy et al., 2002), implying that factors additional to morphological compartmentation contributed appreciably to the diffusion environments detected by MRI in the brain. More recently, the slow diffusion phase (SDP), containing the water molecules with slow diffusion coefficient $\left(D_{\text {slow }}\right)$, has been suggested to represent dynamically restricted water molecules associated with membranes and cytoskeleton structures. Conversely, the fast diffusion phase (FDP), containing the water molecules with a fast diffusion coefficient $\left(D_{\text {fast }}\right)$, has been proposed to represent the remaining, freely moving, solvent water molecules (Niendorf et al., 1996) (Figure 4, upper panels). On these grounds, increases in SDP detected by MRI upon neural activation (Le Bihan, 2007) reveal a relative increment of water molecules diffusing in the vicinity of the membrane and cytoskeleton structures. This is consistent with the increments in monovalent and divalent cation transport occurring during neural activation, thought to occurr initially in the vicinity of the neural membranes. The redistribution may also reflect the increment of neuroglial volume reported to occur during neuronal activation (Andrew and MacVicar, 1994; Hertz et al., 2007; Le Bihan, 2007). In our case, the increase in SDP detected during fasting, might reflect the osmotic swelling of glial cells in the vicinity of stimulated orexigenic synapses, as expected to occur during glutamatergic neurotransmission (Hansson et al., 2000; Simard and Nedergaard, 2004) (Figure 4, lower panels).

< Figure 4, near here> 
More specifically, under fasting conditions, excess glutamate released to the orexigenic cleft is recaptured by surrounding astrocytes, by $\mathrm{Na}^{+}$dependent cotransport mainly through the GLAST/EAAT1 and GLT-1/EAAT2 transporters, with a $3 \mathrm{Na}^{+}$per glutamate stoichiometry (Anderson and Swanson, 2000). The three sodium ions incorporated in this way, are extruded to the extracellular space, in exchange with two potassium ions through the $\mathrm{Na}^{+} / \mathrm{K}^{+}$ATPase. Astrocytic ATP required for the operation of the $\mathrm{Na}^{+} / \mathrm{K}^{+}$ATP-ase and glutamine synthesis during activation by fasting, is thought to be derived from increased glucose consumption and metabolism by oxidative and glycolitic pathways (Pellerin and Magistretti, 1994, Violante et al., 2009) . This increased metabolic demand results in an increased hypothalamic microvascular blood flow, a circumstance consistent with the changes detected here by SPM and LDA under low b weightings (Figure 2D). Additional $\mathrm{K}^{+}$ions accumulated in the extracellular space during orexigenic firing, may enter the astrocyte by stimulation of the $\mathrm{Na}^{+} / \mathrm{K}^{+} / 2 \mathrm{Cl}^{-}$cotransporter (Hertz et al., 2007). Indeed, increased $\mathrm{K}^{+}$concentrations are known to be tightly coupled to neuronal activation, and have been detected using metallographic microscopic imaging approaches (Goldschmidt et al., 2004). Taken together, these processes lead to intracellular potassium accumulation, thus triggering a concomitant water influx and volume increase of the astrocytes, mainly mediated through the highly abundant aquaporin AQP-4 (Badaut et al., 2002). The osmotic swelling response associated to orexigenic stimulation, is proposed to occur initially in few astrocytes, those surrounding the orexigenic cleft, but can be rapidly extended to a plethora of neighbouring astrocytes, through the interconnecting gap junctions of the network arrangement (Halassa and Haydon). This increases significantly the spatial distribution of the orexigenic activation and makes it MRI detectable. Finally, the osmotic swelling response associated to hypothalamic fasting appears not to be isotropic, being mainly detectable in the A$\mathrm{P}$ and L-R directions, suggesting smaller structural restrictions to volume increase in those directions.

In summary, our results show that hypothalamic activation by fasting in mice or humans can be detected non-invasively by Diffusion Weighted Magnetic Resonance Imaging through changes in the water diffusion parameters. The present findings may prove useful for the diagnosis of appetite disorders and can be extended easily to other hypothalamic activation dysfunctions. 


\section{Bibliography}

Anderson CM, Swanson RA (2000) Astrocyte glutamate transport: review of properties, regulation, and physiological functions. Glia 32:1-14.

Andrew RD, MacVicar BA (1994) Imaging cell volume changes and neuronal excitation in the hippocampal slice. Neuroscience 62:371-383.

Badaut J, Lasbennes F, Magistretti PJ, Regli L (2002) Aquaporins in brain: distribution, physiology, and pathophysiology. J Cereb Blood Flow Metab 22:367-378.

Carnell S, Gibson C, Benson L, Ochner CN, Geliebter A (2011) Neuroimaging and obesity: current knowledge and future directions. Obes Rev 13:43-56.

Coll AP, Farooqi IS, O'Rahilly S (2007) The hormonal control of food intake. Cell 129:251-262.

Delgado TC, Violante IR, Nieto-Charques L, Cerdan S (2011) Neuroglial metabolic compartmentation underlying leptin deficiency in the obese ob/ob mice as detected by magnetic resonance imaging and spectroscopy methods. J Cereb Blood Flow Metab 31:2257-2266.

Flint J, Hansen B, Vestergaard-Poulsen P, Blackband SJ (2009) Diffusion weighted magnetic resonance imaging of neuronal activity in the hippocampal slice model. Neuroimage 46:411-418.

Garcia-Martin ML, Ballesteros,P., Cerdan,S. (2001) The metabolism of water in cells and tissues as detected by NMR methods. Progress in Nuclear Magnetic Resonance Spectroscopy 39:41-77.

Gautier JF, Chen K, Salbe AD, Bandy D, Pratley RE, Heiman M, Ravussin E, Reiman EM, Tataranni PA (2000) Differential brain responses to satiation in obese and lean men. Diabetes 49:838-846.

Goldschmidt J, Zuschratter W, Scheich H (2004) High-resolution mapping of neuronal activity by thallium autometallography. Neuroimage 23:638-647.

Hansson E, Muyderman H, Leonova J, Allansson L, Sinclair J, Blomstrand F, Thorlin T, Nilsson M, Ronnback L (2000) Astroglia and glutamate in physiology and pathology: aspects on glutamate transport, glutamate-induced cell swelling and gap-junction communication. Neurochem Int 37:317-329.

Hastie T, Tibshirani R, Friedman J (2001) The elements of statistical learning: Data Mining, Inference and Prediction. New York: Springer Series in Statistics.

Hertz L, Peng L, Dienel GA (2007) Energy metabolism in astrocytes: high rate of oxidative metabolism and spatiotemporal dependence on glycolysis/glycogenolysis. J Cereb Blood Flow Metab 27:219-249.

Le Bihan D (2003) Looking into the functional architecture of the brain with diffusion MRI. Nat Rev Neurosci 4:469-480.

Le Bihan D (2007) The 'wet mind': water and functional neuroimaging. Phys Med Biol 52:R5790.

Le Bihan D (2008) Intravoxel incoherent motion perfusion MR imaging: a wake-up call. Radiology 249:748-752.

Le Bihan D, Urayama S, Aso T, Hanakawa T, Fukuyama H (2006) Direct and fast detection of neuronal activation in the human brain with diffusion MRI. Proc Natl Acad Sci U S A 103:8263-8268.

Liu T, Kong D, Shah BP, Ye C, Koda S, Saunders A, Ding JB, Yang Z, Sabatini BL, Lowell BB (2012) Fasting Activation of AgRP Neurons Requires NMDA Receptors and Involves Spinogenesis and Increased Excitatory Tone. Neuron 73:511-522.

Mahankali S, Liu Y, Pu Y, Wang J, Chen CW, Fox PT, Gao JH (2000) In vivo fMRI demonstration of hypothalamic function following intraperitoneal glucose administration in a rat model. Magn Reson Med 43:155-159. 
Matsuda M, Liu Y, Mahankali S, Pu Y, Mahankali A, Wang J, DeFronzo RA, Fox PT, Gao JH (1999) Altered hypothalamic function in response to glucose ingestion in obese humans. Diabetes 48:1801-1806.

Morton GJ, Cummings DE, Baskin DG, Barsh GS, Schwartz MW (2006) Central nervous system control of food intake and body weight. Nature 443:289-295.

Niendorf T, Dijkhuizen RM, Norris DG, van Lookeren Campagne M, Nicolay K (1996) Biexponential diffusion attenuation in various states of brain tissue: implications for diffusion-weighted imaging. Magn Reson Med 36:847-857.

Parkinson JR, Chaudhri OB, Bell JD (2009) Imaging appetite-regulating pathways in the central nervous system using manganese-enhanced magnetic resonance imaging. Neuroendocrinology 89:121-130.

Paxinos G, Franklin, K.B.J. (2001) The Mouse Brain in Stereotaxic Coordinates. New York Academic Press.

Sehy JV, Ackerman JJ, Neil JJ (2002) Evidence that both fast and slow water ADC components arise from intracellular space. Magn Reson Med 48:765-770.

Simard M, Nedergaard M (2004) The neurobiology of glia in the context of water and ion homeostasis. Neuroscience 129:877-896.

Spanswick DC, Simonds SE, Cowley MA Transmitter Time: Synaptic Plasticity and Metabolic Memory in the Hypothalamus. Cell Metab.

Tataranni PA, Gautier JF, Chen K, Uecker A, Bandy D, Salbe AD, Pratley RE, Lawson M, Reiman EM, Ravussin E (1999) Neuroanatomical correlates of hunger and satiation in humans using positron emission tomography. Proc Natl Acad Sci U S A 96:4569-4574.

van den Pol AN, Wuarin JP, Dudek FE (1990) Glutamate, the dominant excitatory transmitter in neuroendocrine regulation. Science 250:1276-1278.

Violante IR, Anastasovska J, Sanchez-Canon GJ, Rodrigues TB, Righi V, Nieto-Charques L, Parkinson JR, Bloom SR, Bell JD, Cerdan S (2009) Cerebral activation by fasting induces lactate accumulation in the hypothalamus. Magn Reson Med 62:279-283.

Yang Y, Atasoy D, Su HH, Sternson SM (2011) Hunger states switch a flip-flop memory circuit via a synaptic AMPK-dependent positive feedback loop. Cell 146:992-1003. 


\section{Figure Legends}

Figure 1. Overview of the methodology. Diffusion weighted images $\left(10<b<1800 \mathrm{~s} \cdot \mathrm{mm}^{-2}\right)$ are acquired consecutively in the same slice, in three diffusion directions A-P, L-R and H-F. The diffusion data set is analyzed with two independent approaches: a biexponential model fit (upper panels) and a Fisher Discriminant Analysis (lower panels). From the biphasic model, coloured maps of the independent parameters fitted are obtained, revealing significant differences in the diffusion parameters (SDP, FDP, $D_{\text {slow }}, D_{\text {fast }}$ ) between the fed and fasted brains. Investigated areas were the hypothalamus (excluding the third ventricle, circled in blue) and the somatosensory cortex (circled in yellow). Using Fisher Discriminant Analysis, the entire diffusion imaging data set from the fed and fasted brains, can be classified successfully $(100 \%$ success) in the two feeding sates: with the most different pixels representing the fed (blue) or fasted (red) pixels.

Figure 2. Imaging appetite in the mouse brain. A: Axial and coronal MRI sections containing the hypothalamus in a representative mouse brain model and in a brain atlas (insert). B (left): Hypothalamic A-P SDP colour maps obtained from the DWI of fed or fasted animals (upper panels), superimposed to the corresponding $T_{2}$-weighted $\left(T_{2 w}\right)$ anatomical brain images. The hypothalamic region is shown enlarged in the lower panels. B (right) Values of hypothalamic SDP, $D_{\text {slow }}$ and $D_{\text {fast }}$ parameters (mean $\pm S D$ ) in the L-R, A-P and H-F directions.. The number of pixels used in the comparisons was: $N_{\text {fed }}=120$ and $N_{\text {fasted }}=141$, in L-R; $N_{\text {fed }}=95$ and $N_{\text {fasted }}=82$ in L-R and $\mathrm{N}_{\text {fed }}=201$ and $\mathrm{N}_{\text {fasted }}=176$ in the H-F directions, respectively. C (left): Cortical areas investigated from a representative mouse brain, in the fed and fasted states (upper images). The A-P SDP values are shown superimposed to anatomical $T_{2 w}$ images. The corresponding enlargements of the cortical areas are shown below. $C$ (right): SDP, $D_{\text {slow }}$ and $D_{\text {fast }}$ values (mean \pm sd) of six mice in the fed and fasted conditions. The number of pixels used for the comparisons are: $\mathrm{N}_{\text {fed }}=7$ and $\mathrm{N}_{\text {fasted }}=21$, in the L-R direction; $\mathrm{N}_{\text {fed }}=31$ and $\mathrm{N}_{\text {fasted }}=32$ in L-R measurements; $\mathrm{N}_{\text {fed }}=57$ and $\mathrm{N}_{\text {fasted }}=71$ in $\mathrm{H}-\mathrm{F}$ orientation. $\mathrm{D}$ (left): SPM analysis of the areas that best differentiate the fed and fasted hypothalamus in a representative mouse brain (left). Note the hypothalamic (yellow arrow) and vascular (blue arrow) activations.D (right): "Appetite Index" histogram of the fed (blue) and fasted (red) pixels as projected over calculated the LDA vector. Note the almost complete resolution of fed and fasted histograms.. Panels on the right 
side illustrate the localization in the mouse brain image of the $5 \%$ lowest (fed) and highest points (fasted) at each end of the appetite index histogram.

Figure 3. Imaging appetite in the human brain. A: Coronal and sagital sections containing the hypothalamus in a representative human brain. B (left) Parameter maps showing the H-F SDP pixel values of the six volunteers in the fed and fasted conditions, superimposed to anatomical $T_{2 w}$ images (upper panels) and enlarged in the lower panels. B (right) Hypothalamic values of SDP, $D_{\text {slow }}$ and $D_{\text {fast }}$ parameters, (mean $\left.\pm S D\right)$ of six human subjects in the fed and fasted conditions. The number of pixels used in the comparisons is: $N_{\text {fed }}=7, N_{\text {fasted }}=4$, in L-R direction; $N_{\text {fed }}=13, N_{\text {fasted }}=12$ in L-R and $N_{\text {fed }}=24, N_{\text {fasted }}=16$ in H-F direction. C (left): Cortical areas analyzed in the human brain, in the fed and fasted conditions (upper panels)., with $\mathrm{H}-\mathrm{F}$ SDP values superimposed to anatomical $T_{2 w}$ images. The corresponding enlargements of the cortical areas are shown below. $C$ (right): SDP, $D_{\text {slow }}$ and $D_{\text {fast }}$ values (mean $\pm s d$ ) The number of pixels used for the comparisons are: $N_{\text {fed }}=55$ and $N_{\text {fasted }}=114$ in the L-R direction; $N_{\text {fed }}=104$ and $N_{\text {fasted }}=106$ in L-R direction; $N_{\text {fed }}=102$ and $N_{\text {fasted }}=83$ in H-F direction. D (left) "Appetite Index" histogram for the fed (blue) and fasted (red) human hypothalamus, calculated using LDA. $\mathrm{D}$ (right) Identification of the areas representing the position of the $5 \%$ of the points located in the lower (fed) and upper (fasted) extremes of the Appetite Index histogram.

Figure 4. Neuroglial metabolic coupling and astrocytic volume changes underlie the orexigenic hypothalamic activation as detected by fDWI. The upper panels illustrate the effects of volume changes in the phase distribution (SDP) and dynamics ( $\left.D_{\text {slow }}\right)$ of water molecules around the plasma membrane, under the fed (left) and fasted (right) conditions. Water molecules close to the plasma membrane (dark blue) experience more restricted diffusional motions than those far apart (light blue). Increases in astrocytic volume (right) produce; (i) an increase in the available membrane surface, augmenting the contribution of diffusionally restricted water molecules (increased SDP, more water molecules in the dark blue area) and (ii) a decrease in the average obstructions allowing faster average water diffusion (increased $D_{\text {slow, }}$ faster diffusion in the light blue area). The lower panels illustrate the neuroglial metabolic coupling mechanisms and associated volume responses in the fed (left) and fasted (right) hypothalamus. During orexigenic neurotransmission, excess glutamate released to the synaptic cleft by AGRP/NPYY neurons (orange) is recaptured by surrounding astrocytes, 
together with $3 \mathrm{Na}^{+}$, through the astrocytic glutamate cotransporters GLAST (grey). Intracellular sodium ions thus incorporated are extruded to the extracellular space, through the electrogenic $\mathrm{Na}^{+} / \mathrm{K}^{+}$ATPase (yellow), incorporating two intracellular $\mathrm{K}^{+}$ions. Astrocytic glutamate produces glutamine, through glutamine synthase, which is later recaptured by the neurons to operate the glutamine cycle. Astrocytic ATP molecules required for glutamine synthesis and the $\mathrm{Na} / \mathrm{K}^{+}$ ATPase, are generated from plasma glucose, after transport through GLUT1 (white), by anaerobic glycolisis and the tricarboxyilic acid cycle. Lactate produced is extruded through the monocarboxylate transporter (MCT1, green) and potentially oxidized by surrounding neurons. Additional $\mathrm{K}^{+}$ions may be incorporated to the astrocyte through the $\mathrm{Na}^{+} \mathrm{K}^{+} 2 \mathrm{Cl}^{-}$cotransporter (purple), resulting in increased intracellular $\mathrm{K}^{+}$concentrations and thus triggering, an osmotically driven, aquaporin 4 (AQP4) mediated (blue channel), water transport and astrocytic swelling. Under fasting conditions (right), orexigenic firing is increased (darker lines right) as well as glutamatergic neurotransmission (increased glutamate in the synaptic cleft), resulting in augmented ionic and water trafficking (darker arrows, more water influx through AQP4). The accumulated $\mathrm{K}^{+}$in one astrocyte may be transferred to neighboring astroglia, through gap junctions (inverted brackets), providing a spreading of the swelling response through the astrocyte syncithium. The central bargraphs represent color coded SDP and $\mathrm{D}_{\text {slow }}$ variations, as detected by fDWI. A: Astrocyte, ARC:, Arcuate, PVN: Paraventricular, MC4, MC3: Melanocortin receptors, N: Neuron, AGRP/NPY: Agouti Related Protein/NeuroPeptideY, POMPCT/CART: Proopiomielanocortin transcript/Cocaine Anfetamine Regulated Transcript. Mechanisms are shown only in one astrocyte for simplicity. 
PARAMETER MAPS
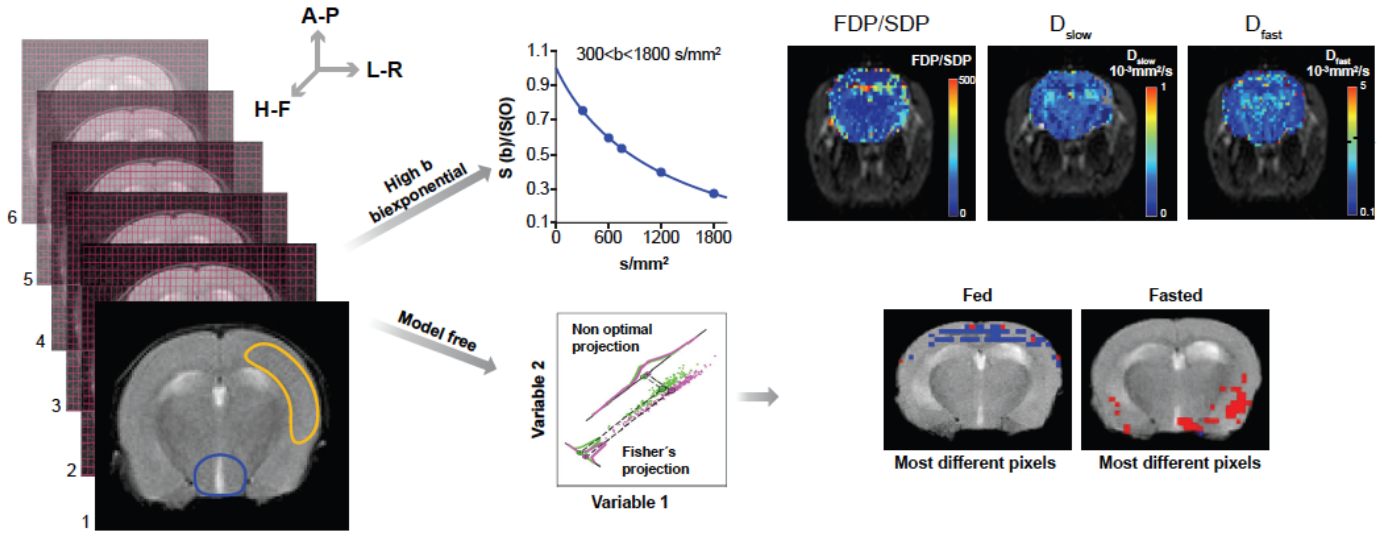

Figure 1.

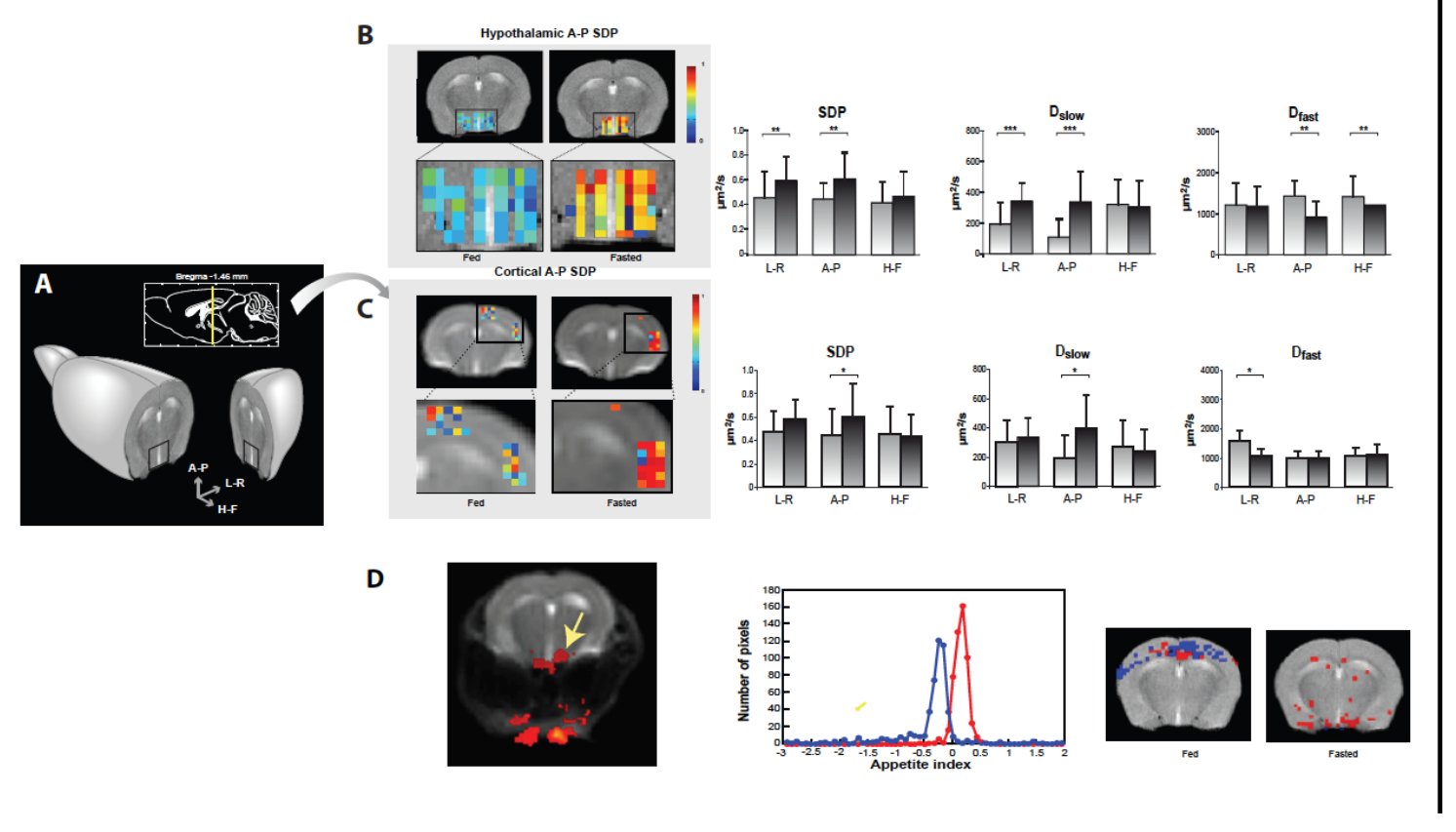


Figure 2.

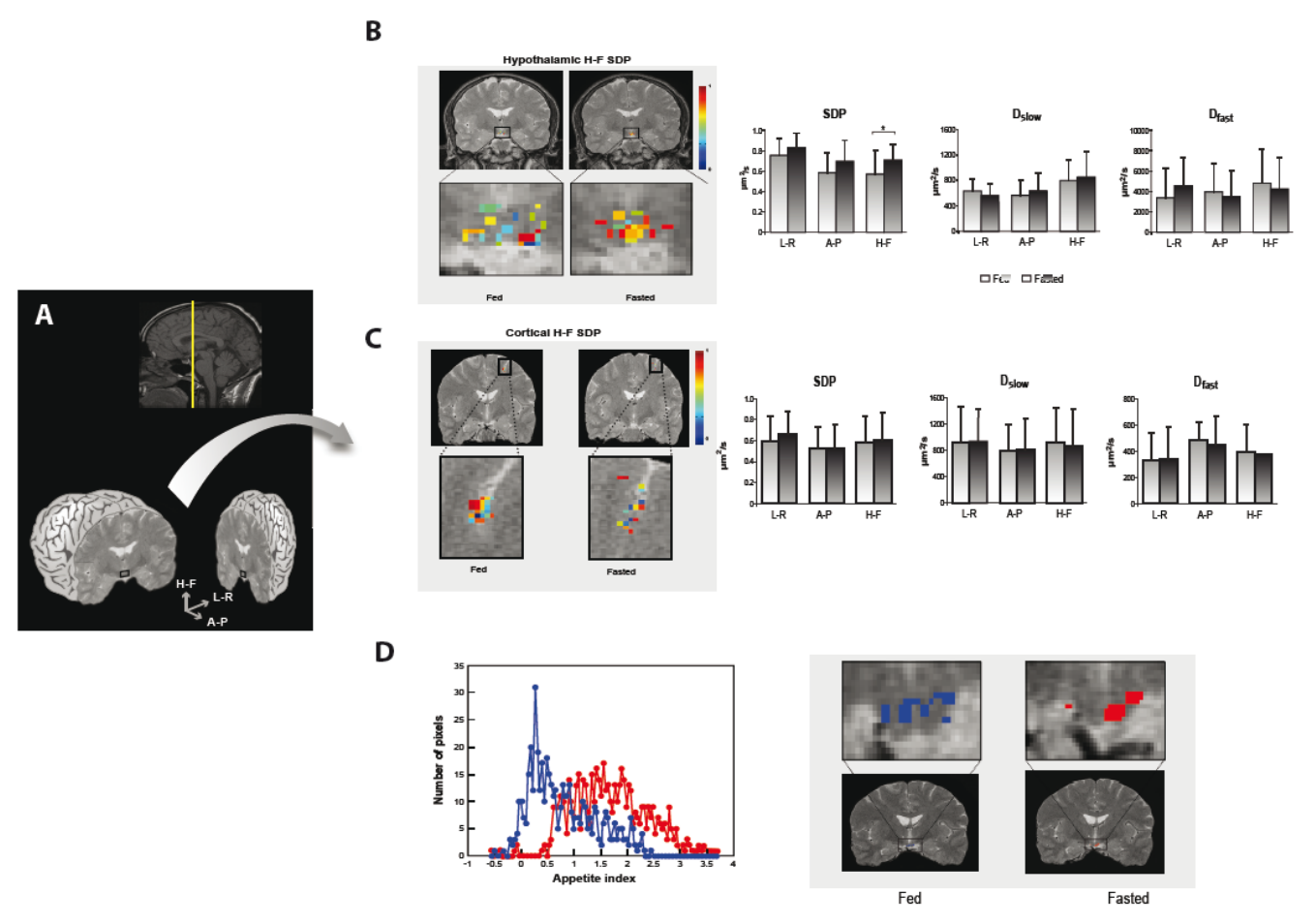


Figure 3.

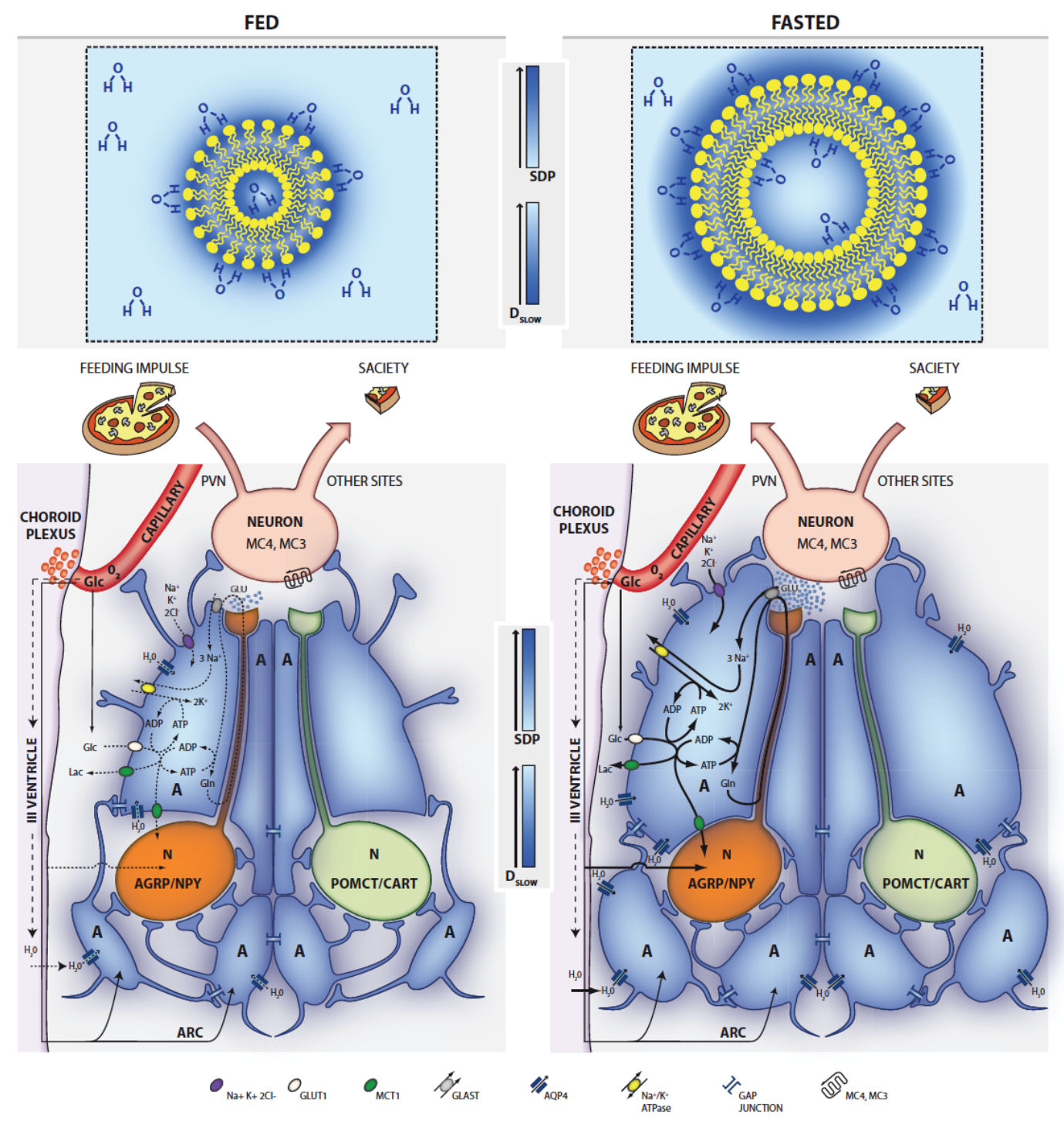


Table 1 Blood concentrations of relevant biochemical parameters of the six volunteers, determined after each imaging session. Mean values of the same parameters in both feeding conditions are also provided, as well as reference values for healthy adult conditions

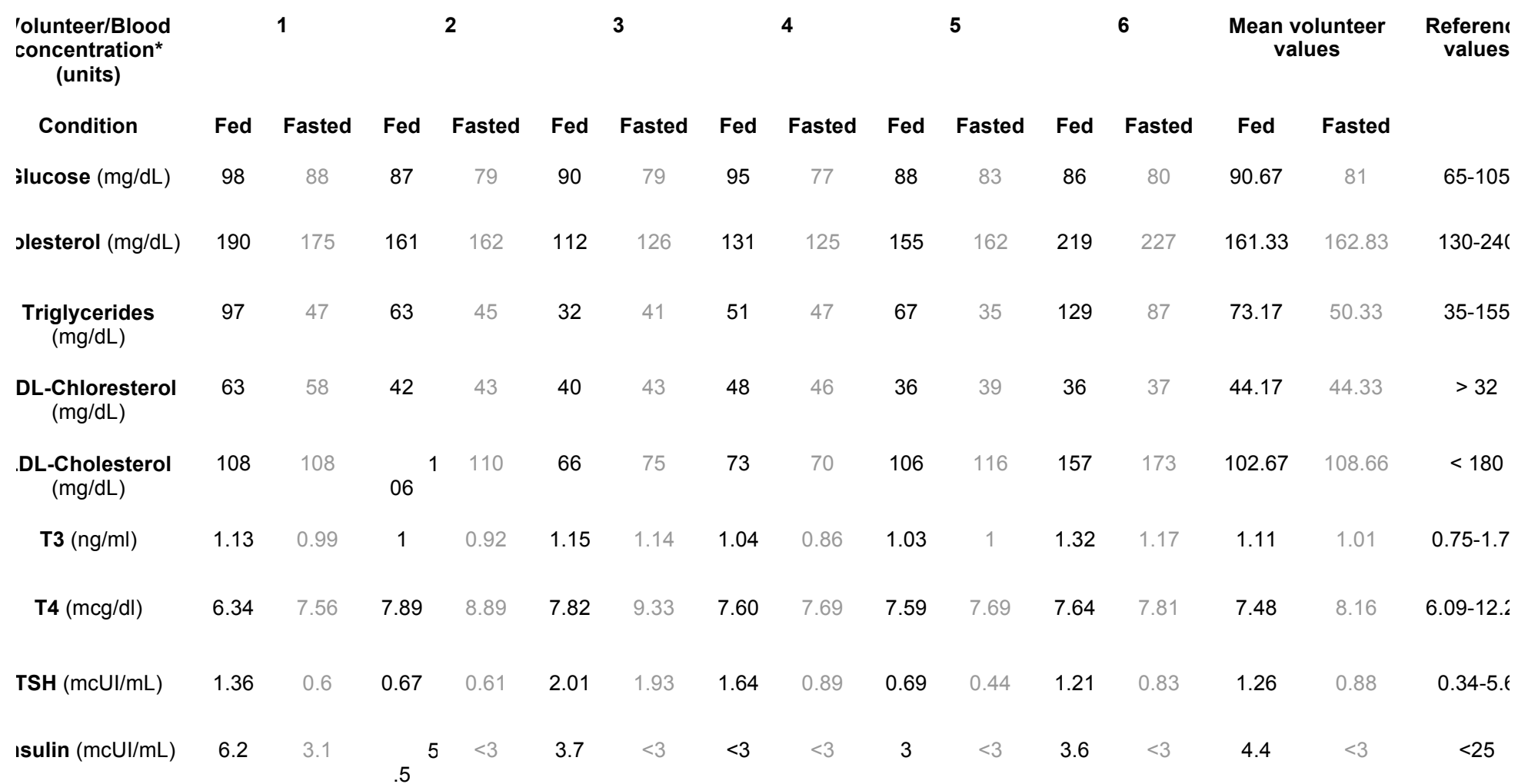

*Determinations were performed using clinically validated protocols by Analitical Biochemistry Laboratory Services of the Clinica Nuestra Sra. Del Rosario (Madrid). Briefly, glucose was measured using the glucose oxidase method (One touch Ultra, Lifescan, Johnson and Johnson, Issy-les-Moulineaux, FR). Total serum cholesterol, triglycerides were measured enzymatically using a CHOD-PAP test (Boehringer- Mannheim, DE), T3, T4 and TSH determination used the Accubind-ELISA kit and insulin with the insulin ELISA kit (Millipore, Billerica, MA, USA). 